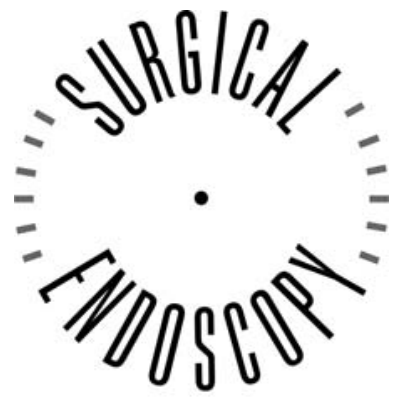

and Other Interventional Techniques

\title{
The mechanical master-slave manipulator: an instrument improving the performance in standardized tasks for endoscopic surgery
}

\author{
J. Diks, ${ }^{1}$ J. E. N. Jaspers, ${ }^{2}$ W. Wisselink, ${ }^{1}$ B. A. M. J. de Mol, ${ }^{3}$ C. A. Grimbergen ${ }^{2}$ \\ ${ }^{1}$ Department of Surgery, VU Medical Center (VUMC), Amsterdam, The Netherlands \\ ${ }^{2}$ Department of Medical Technological Development, Academic Medical Center (AMC), Meibergdreef 9, 1105 AZ, Amsterdam, The Netherlands \\ ${ }^{3}$ Department of Cardio Thoracic Surgery, Academic Medical Center (AMC), Meibergdreef 9, 1105 AZ, Amsterdam, The Netherlands
}

Received: 31 March 2006/Accepted: 27 April 2006/Online publication: 6 February 2007

\begin{abstract}
Background: This study aimed to evaluate the feasibility and efficacy of a mechanical minimally invasive manipulator for endoscopic surgery. In contrast to currently available motorized master-slave manipulators, this mechanical manipulator consists of two purely mechanical, hand-controlled endoscopic arms with joints that allow seven degrees of freedom (DOF).

Methods: For the study, 30 medical students performed four different tasks in a pelvic trainer box using either two conventional endoscopic needleholders or a set of mechanical manipulators. The exercise consisted of four different tasks: repositioning of coins, rope passing, passing of a suture through rings, and tying of a surgical knot. All experiments were recorded on videotape (S-VHS), and the data were analyzed afterwards by an independent observer using a quantitative time-action analysis.

Results: A significant difference in the number of total actions (including failures) favoring the mechanical manipulator group was shown in most exercises. A significant difference in failures per task was shown in favor of the mechanical manipulator group as well. There was no significant difference shown in the total time per exercise.

Conclusions: The tasks clearly demonstrated the efficacy of the mechanical manipulator, although some technical flaws emerged during the experiments. Considering the fact that a first prototype of the mechanical manipulator was tested, modifications are to be expected in a next model. These experiments show the potential of the mechanical manipulator, and it is expected to be a competitive and economical instrument for endoscopic surgery in the near future.
\end{abstract}

Correspondence to: J. E. N. Jaspers
Key words: Master-slave surgery - Surgical skills Mechanical manipulator - Minimally invasive manipulator

The purpose of endoscopic surgery is to reduce surgical trauma to patients, resulting in less operative morbidity, faster recovery, and reduction in costs $[1,17]$. The design of endoscopic instruments, based initially on conventional surgical tools, are long with only four degrees of freedom (DOF) in positioning (Fig. 3). These straight instruments have to pivot about a point of incision through the abdominal wall, which introduces a mirroring and a variable scaling of the hand movements controlling the tip of the instrument. The surgeon must compensate for this scaling and fulcrum effect [5].

With conventional endoscopic instruments and their limited number of DOF for positioning, it is impossible to approach the tissue from different directions (Fig. 1), and almost impossible to perform delicate and complex surgical actions. The handles of these long instruments force the surgeon to use his hands in an unsupported and unnatural posture with a large distance between both hands. The ergonomic quality of laparoscopic instruments is relatively poor $[3,20]$. Due to the length and the orientation of these instruments, the surgeon often must operate in an uncomfortable posture with extreme wrist positions.

Furthermore, vision in laparoscopic surgery is twodimensional. The image of an endoscopic camera is projected on a monitor. Largely because of these characteristics, the learning curves for minimally invasive surgery are long and steep [11, 19]. Especially for complex procedures, the applicability of minimally invasive surgery has not yet been widely embraced.

Recently introduced robotic surgical systems have facilitated complex endoscopic surgery, such as micro- 


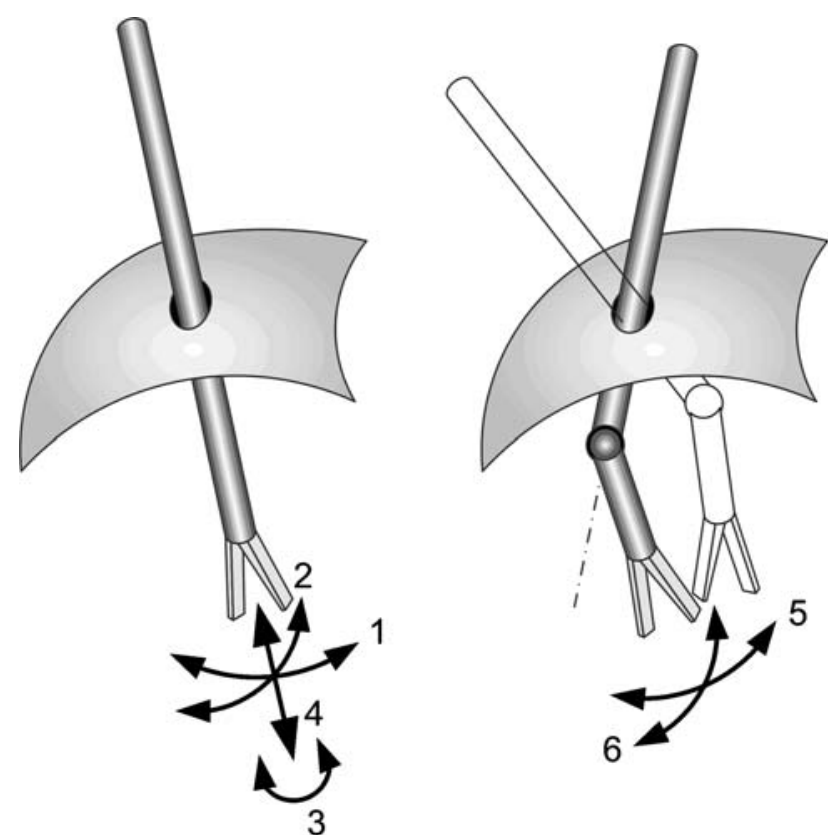

Fig. 1. Schematic master-slave system. The instrument tip and the surgeon's handle has six degrees of freedom in positioning. With a kinematic coupling between the handle and the tip, the instrument tip moves exactly in the same direction as the handle. This kinematic link can be implemented electronically (using sensors, actuators, and the like), as with robotic devices, or mechanically (using push bars, pulleys, and the like), as with a mechanical manipulator (picture by Mark Wentink).

anastomoses in coronary artery bypass grafting [10, 12] and aortic anastomosis $[14,22]$. These systems are designed to translate the surgeon's hand movements to the tip of the endoscopic instrument in a remote operative field using a computer-assisted master-slave system (Fig. 2). The advantages of these systems are threedimensional visualization and inclusion of a "wrist" at the end of the instruments, providing articulated motion in seven DOF: three translations, three rotations, and the opening/closing action [7]. The wrist movements of the surgeon's hands are translated to the movements of the instrument tip, maintaining the same spatial relation.

Although it seems that these robotic surgical systems have their advantages, and although various series are reported in which these systems have been successfully applied for clinical purposes $[6,18]$, their disadvantages are not to be taken lightly. These systems are large, bulky, and expensive. Another limitation of these systems is the lack of force feedback $[2,4,15]$. The feedback from the operation field consists merely of visual information. Lowering the costs and making the system more manageable are mandatory if these systems are to become a standard tool for endoscopic surgery.

In an attempt to overcome some of the aforementioned limitations of robotic systems, a mechanical master-slave manipulator for minimally invasive surgery was developed. The mechanical manipulator is a relatively small, economical, and mechanical alternative for robotic surgical systems with the additional value of force feedback. It consists of a balanced parallelogram mechanism with a deflectable endoscopic instrument attached at one end and a surgeon's handle at the other end. Instead of an electrical link, the instrument and handle are connected by a mechanical link such that movement directions of the handle correspond to identical movement directions of the instrument tip in seven DOF. In addition, with a set of these devices, the two handles have the same spatial orientation in relation to each other as the instrument tips. As compared with conventional endoscopic instruments, the mechanical manipulator improves the ergonomics for the surgeon, enabling a positioning of his or her hands in a natural orientation to each other, providing improved eye-hand coordination, intuitive manipulation, and an ergonomic posture (Figs. 2 and 3).

The first phantom experience indicated that the system functions properly, and that suturing is feasible [9]. We hypothesised that working with the mechanical manipulator would be more intuitive, and that the extra DOF would offer advantages over conventional endoscopic instruments. To test these hypotheses, we defined simple and reproducible manipulation experiments in which these extra functionalities would play a role. These experiments were used subsequently to compare manipulation between working with conventional endoscopic instruments and working with the mechanical manipulator.

\section{Methods and materials}

For this study, 30 medical students, all with no surgical experience, performed four different experiments in a trainer box. Defined actions and failures per experiment are presented in Table 1. The participants were randomized to perform the experiments with either two conventional endoscopic needleholders (Karl Storz, Tuttlingen, Germany) or a set of mechanical manipulators.

To exclude bias, both setups were identical. The trainer boxes were identical, and all experiments were performed with the instruments positioned in the same orientation to the target area for both groups. The endoscope, positioned in a holder (PASSIST) [8] between the instruments and parallel to the surgeon's natural line of sight [21], was not controlled during the experiments. A $10-\mathrm{mm} 0^{\circ}$ stereoscopic endoscope and three-dimensional camera (Carl Zeiss Ltd., Oberkochen, Germany) in combination with a Cardio View Head-Up-Display (VISTA Medical Technologies, Inc., Carlsbad, CA, USA) (Fig. 3) was used in all the experiments to provide the subjects with a stereoscopic image, claimed to be beneficial in using instruments with additional DOF [7].

Due to the simple nature of the tasks, the participants were not allowed to rehearse the exercises before the experiments. They were, however, allowed a 1-min period to familiarize themselves with the setup $[7,13,16]$.

\section{Experiment 1: Coin repositioning}

For this simple pick and place experiment, a 1-Eurocent coin had to be taken out of a receptacle with the left-hand instrument and presented to the right-hand instrument. Subsequently, the subject was to put the coin into a second receptacle. This sequence of picking up, passing over, and dropping was repeated two times. The same order of sequences then was repeated, starting with the right-hand instrument. Unintentional or incorrect dropping of a coin was counted as a failure. The number of defined actions was counted, and the total time was recorded from picking up the first coin to dropping the last coin into the last receptacle. 


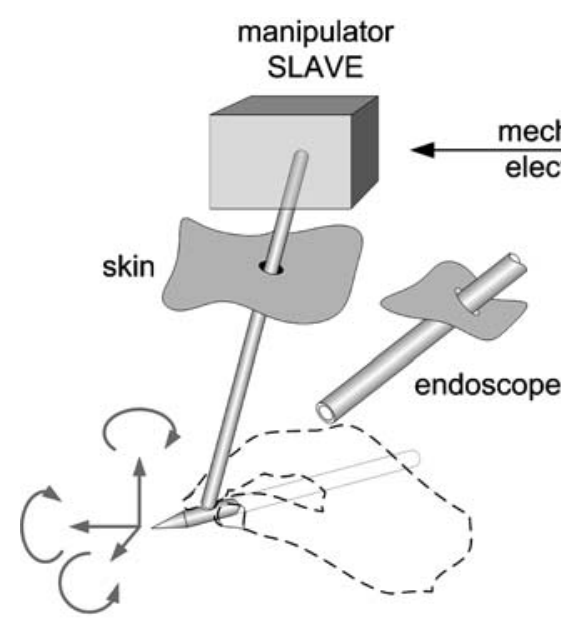

PATIENT surgeon console MASTER

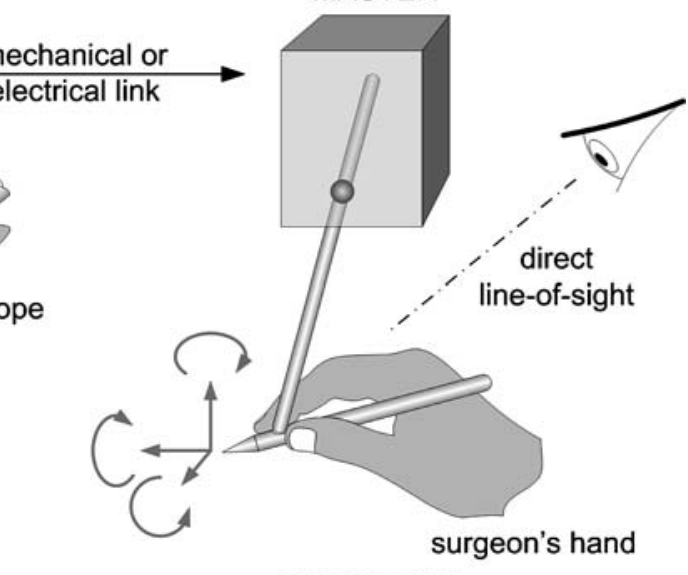

SURGEON
Fig. 2. The mechanical manipulator in the experimental setup. The picture shows two parallelogram mechanisms coupled to the table rail. The inset shows that movement directions of the handle correspond to identical movement directions of the instrument tip in all degrees of freedom.

Table 1. Defined actions and failures

\begin{tabular}{|c|c|}
\hline Actions/failures: & Definitions \\
\hline \multicolumn{2}{|l|}{ Coins } \\
\hline Grasping coin & Grasping a coin \\
\hline Lifting coin & Elevating coin from receptacle \\
\hline Transferring coin & Moving coin toward next receptacle \\
\hline Handing over coin & Handing coin over from one instrument to the other \\
\hline Dropping coin & Dropping coin correctly into receptacle \\
\hline Unintentionally dropping coin & Unintentional dropping coin during exercise \\
\hline Incorrectly dropping coin & Dropping coin outside the receptacle or into the wrong receptacle \\
\hline \multicolumn{2}{|r|}{11} \\
\hline Grasping rope & Grasping the rope at the correct place \\
\hline Dropping rope & Dropping rope \\
\hline Misgrasping rope (out of place) & Grasping rope, but at the wrong place \\
\hline Misgrasping rope (no rope) & Grasping without touching rope \\
\hline \multicolumn{2}{|l|}{ Rings } \\
\hline Grasping needle & Each grasping of the needle or suture during exercise \\
\hline Passing ring & Passing a ring \\
\hline Dropping needle & Dropping the needle during exercise \\
\hline Floating needle & Dropping needle while passing ring; needle does not fall, but hangs in the ring \\
\hline Misgrasping & Grasping without touching the needle \\
\hline Wrong direction & Passing a ring in wrong direction \\
\hline Missing ring & Moving toward ring without passing it \\
\hline Half passing & Passing the ring halfway and subsequently taking the needle back from the ring \\
\hline \multicolumn{2}{|r|}{ - } \\
\hline Making loop & Making a loop with the suture \\
\hline Grasping suture & Grasping the suture \\
\hline Pulling through & Pulling the suture through the loop \\
\hline Mislooping & Making a loop without success \\
\hline Misgrasping & Grasping without touching the suture \\
\hline Mispulling & Pulling the suture without passing the loop \\
\hline
\end{tabular}

\section{Experiment 2: Rope passing}

In this experiment, the two instruments had to work together during the manipulation. The subject had to grasp a marked rope $(25 \times 0.3$ $\mathrm{cm}$ ) alternately with the left and the right instruments at indicated points while keeping the rope above the floor of the training box. The rope was fastened at both ends and had 11 predetermined, marked grasping points. The subject had to pass the rope through twice, once grasping the rope with both instruments on the same (left) side and once grasping the rope on both sides, on the right side with the right instrument and on the opposite (left) side with the left instrument. Grasping without touching the rope, grasping the printed lines between the marked areas, or dropping the rope was counted as a failure. Time from picking up the rope to total runthrough was recorded, and the number of defined actions was counted (Fig. 4).

\section{Experiment 3: Passing a suture through rings}

The purpose of this task was to pass a surgical needle with a piece of suture (Prolene 4-0) through eight rings following a preindicated direction. Failures were determined as dropping the needle, "floating" the needle in a ring without grasping it, grasping without touching the needle, not following the indicated direction, missing the ring with the needlepoint, and passing the ring only halfway. The total number of actions and the time from first grasping the needle to totally passing the last ring was recorded (Fig. 5).

\section{Experiment 4: Tying a surgical knot}

A suture (Vicryl 3-0) was used to tie a surgical knot consisting of one knot using two forward loops followed by one knot using a backward 


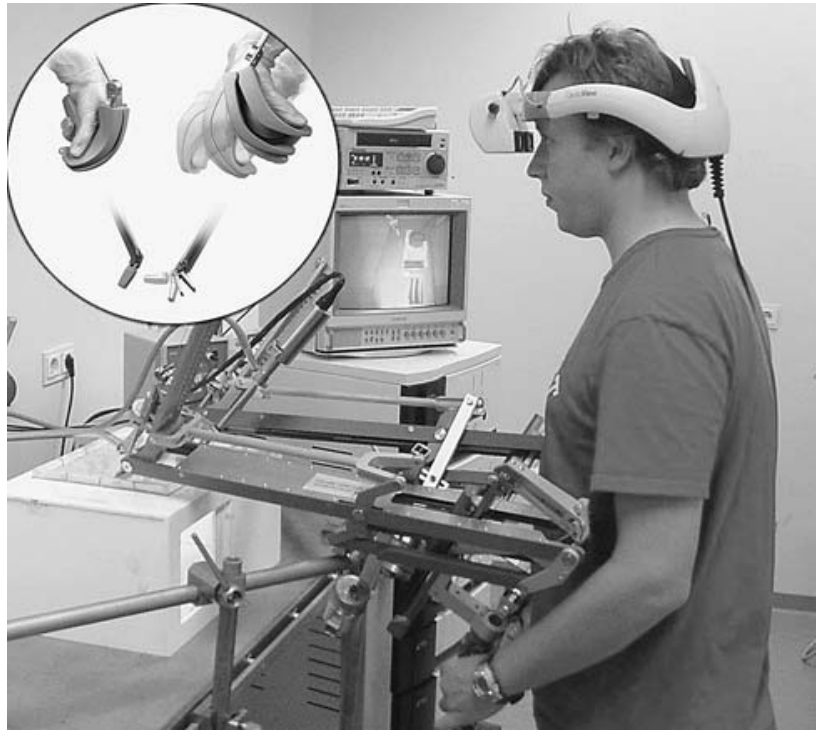

Fig. 3. Conventional endoscopic instruments (left) have four degrees of freedom (DOF): two rotations around the incision in the skin (DOF 1 and 2), the rotation of the instrument around its axis (DOF 3), and the in-out translation of the instrument (DOF 4). By adding two extra DOF (right) (DOF 5 and 6), the orientation of the instrument tip can be varied independently from the instrument shaft, enabling the surgeon to approach the tissue from different directions. The opening and closing of the instrument is the seventh DOF (picture by Mark Wentink).

loop. The scored failures were "mislooping" the suture, grasping without touching the suture, and pulling the suture without passing the loop. The total number of actions and the total time needed to complete the task were recorded.

\section{Statistical analysis}

All the experiments were recorded on videotape (S-VHS), and the data were analysed afterward by an independent observer using a quantitative time-action analysis to determine the efficacy, counting the actions and the time needed per task. Failures were counted as a measure for efficiency. A quantitative time-action analysis was performed, through which a measure of efficiency in time and actions was determined. Table 1 shows the definitions for actions and failures per experiment.

Statistical analysis was performed using SPSS 12.0.1 for Windows. A Mann Whitney $U$ test was used to compare differences between the two methods. The data show medians of time and actions with the corresponding range. A $p$ value less than 0.05 was considered statistically significant.

\section{Results}

All the participants successfully completed the experiments, although six participants in the mechanical manipulator group had problems with the knot-tying experiment (see Discussion section).

Tables 2 and 3 show the results of the time-action analysis. Table 2 shows the median time and range needed per experiment for completion of the task. There was no statistically significant difference shown in the time needed to complete each exercise. In Table 3, median actions and the range needed per experiment are shown per action, as well as the median failures and

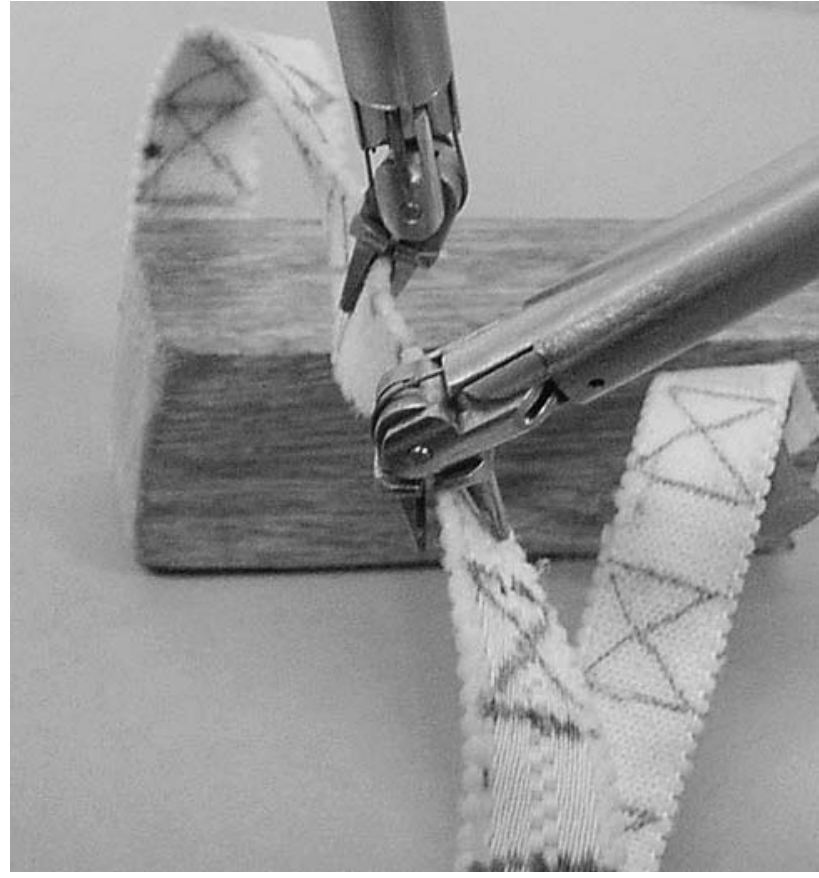

Fig. 4. Experiment 2: Band-passing, manipulating a marked band with two instrument by grasping it at the same side (minimally invasive manipulator experiment).

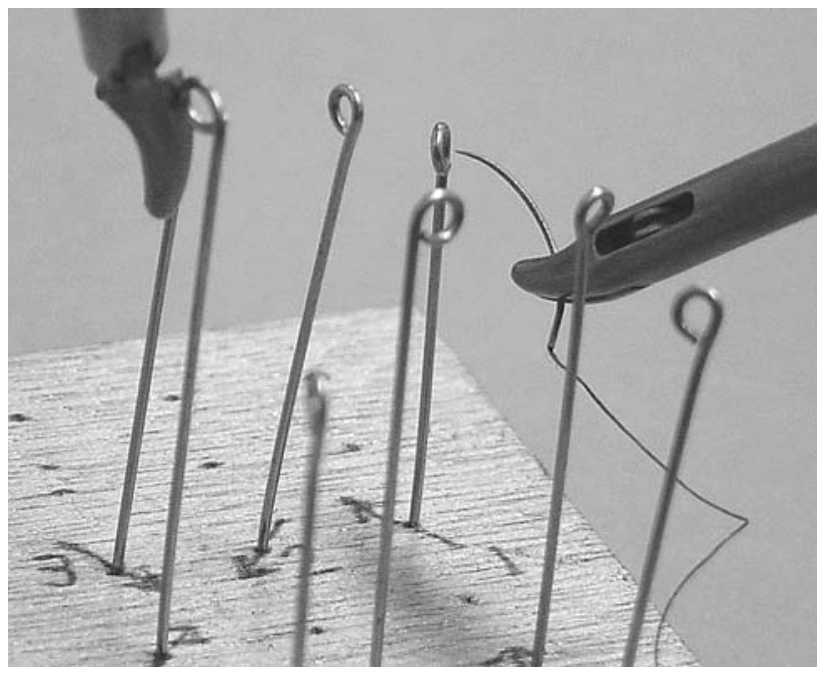

Fig. 5. Experiment 3: Passing a suture through rings, following a predefined path (laparoscopic experiment).

range per experiment. Table 3 and Fig. 6 show the median total of actions (including failures) and the range needed per experiment.

There were significantly fewer actions recorded in the mechanical manipulator group for all exercises except the knot-tying experiment. Subanalysis of the different exercises showed that grasping actions were significantly fewer in the mechanical manipulator group for the coin exercise (experiment 1: median, 7 [range, 6-13] vs median, 10 [range, $6-18$ ]; $p=0.01$ ) and the rings exercise (experiment 3: median, 30 [range, 25-45] vs median, 38 [range, 22-99]; $p=0.03$ ). Failures were shown to be 
Table 2. Time (seconds) per exercise necessary to complete exercise (median)

\begin{tabular}{|c|c|c|c|c|c|}
\hline & \multicolumn{2}{|c|}{ Laparoscopy $(n=15)$} & \multicolumn{3}{|c|}{ Mechanical manipulator $(n=15)$} \\
\hline & Time & Range & Time & Range & $p$ Value \\
\hline Coins & 301 & $126-622$ & 339 & $151-600$ & NS \\
\hline Rope & 393 & $183-890$ & 349 & $144-581$ & NS \\
\hline Rings & 704 & $407-1,320$ & 814 & $506-1,529$ & NS \\
\hline Knot & 211 & $68-804$ & $237(n=9)$ & $128-1,395$ & NS \\
\hline
\end{tabular}

NS, not significant

Table 3. Number of actions necessary to complete exercise (median)

\begin{tabular}{|c|c|c|c|c|c|}
\hline & \multicolumn{2}{|c|}{ Laparoscopy $(n=15)$} & \multicolumn{3}{|c|}{ Mechanical manipulator $(n=15)$} \\
\hline & Actions $(n)$ & Range & Actions $(n)$ & Range & $p$ Value \\
\hline \multicolumn{6}{|l|}{ Coins } \\
\hline Grasping coin & 10 & $6-18$ & 7 & $6-13$ & 0.01 \\
\hline Lifting coin & 9 & $6-16$ & 6 & $6-13$ & 0.01 \\
\hline Transferring coin & 8 & $6-16$ & 6 & $6-13$ & 0.03 \\
\hline Handing over coin & 9 & $6-18$ & 6 & $6-14$ & 0.04 \\
\hline Dropping coin & 6 & $6-6$ & 6 & $6-6$ & NS \\
\hline Failures & 5 & $1-12$ & 1 & $0-8$ & $<0.001$ \\
\hline Total (including failures) & 48 & $32-86$ & 32 & $30-67$ & $<0.001$ \\
\hline \multicolumn{6}{|l|}{ Rope } \\
\hline Grasping rope & 22 & $20-36$ & 21 & $21-23$ & NS \\
\hline Failures & 10 & $3-29$ & 1 & $0-8$ & $<0.001$ \\
\hline Total (including failures) & 35 & $24-64$ & 23 & $21-31$ & $<0.001$ \\
\hline \multicolumn{6}{|l|}{ Rings } \\
\hline Grasping needle & 38 & $22-99$ & 30 & $25-45$ & 0.03 \\
\hline Passing ring & 8 & $8-8$ & 8 & $8-8$ & NS \\
\hline Failures & 21 & $10-101$ & 16 & $5-31$ & NS $(0.068)$ \\
\hline Total (including failures) & 67 & $44-208$ & 55 & $40-82$ & 0.02 \\
\hline Knot & & & $(n=9)$ & & \\
\hline Making loop & 6 & $3-10$ & 6 & $3-21$ & NS \\
\hline Grasping suture & 2 & $2-6$ & 2 & $1-15$ & NS \\
\hline Pulling through & 2 & $2-2$ & 2 & $0-3$ & NS \\
\hline Failures & 6 & $0-20$ & 3 & $0-35$ & NS \\
\hline Total (including failures) & 15 & $7-35$ & 10 & $6-73$ & NS \\
\hline
\end{tabular}

NS, not significant

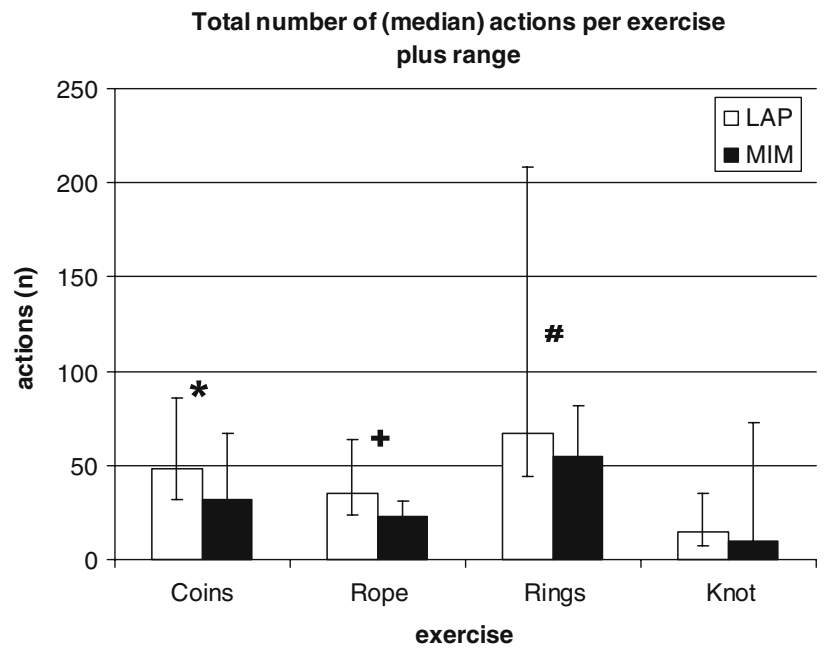

Fig. 6. Total number of (median) actions per exercise plus range.

significantly fewer in the mechanical manipulator group for the coin exercise (experiment 1: median, 1 [range, 0 8] vs median, 5 [range, 1-12]; $p<0.001$ ) and rope exercise (experiment 2: median, 1 [range, $0-8$ ] vs median, 10 [range, 3-29]; $p<0.001)$. In the rings and the knot experiments, no significant difference in failures was shown, although a trend in the rings experiment was shown in favor of the mechanical manipulator group (median, 16 [range, 5-31] vs median, 21 [range, 10-101]; $p=0.068)$.

\section{Discussion}

The experiments were designed as simple tasks that could be executed in both groups, mainly due to the level of our participants' inexperience. They had to carry out the experiments with no surgical/endoscopic experience whatsoever to prevent a bias in the learning curve attributable to experience in either laparoscopy or robotic surgery. The experiments, however, were representative and resembled experiments used in the residents program for endoscopic surgery. Furthermore, we selected a larger number of participants than similar studies $[7,13]$ to exclude bias attributable to eye-hand 


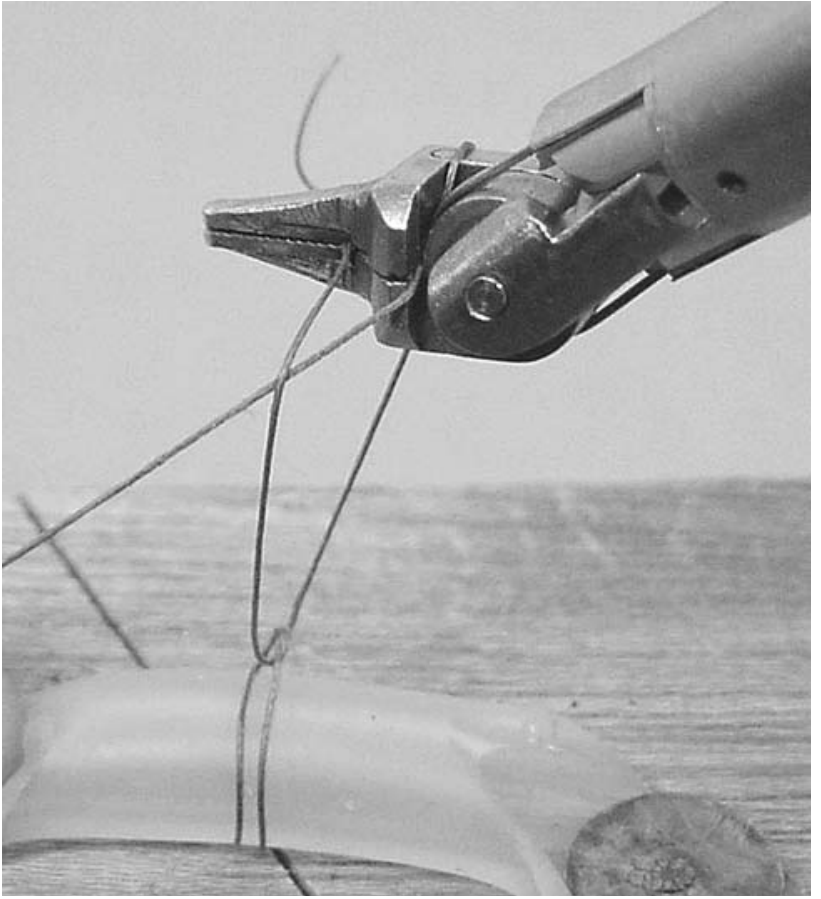

Fig. 7. Experiment 4: Knot tying. A complication in this exercise is that with the minimally invasive instruments, the suture is caught in the grasper mechanism.

coordination. The range in time needed to complete the exercise (Table 2) shows that we succeeded in compiling homogeneous groups in this respect.

The set of mechanical manipulators was a first set of prototypes, and therefore was technically not yet perfected. During testing, technical flaws emerged, which will be corrected in a next prototype. It was noted that the handles were not positioned quite favorably, resulting in an additional number of failures, such as dropping of the needle in experiment 3. Furthermore, one of the mechanical manipulators showed excessive friction in one DOF (in-out), which made it harder to manipulate in a small range, as in experiment 3.

In the knot-tying experiment, another flaw of design was noted. Six participants had trouble sliding a loop from one of the instruments. The joints at the tip of the instruments were not protected in the design, making it an easy trap in which a piece of suture could get caught. This happened in 6 of the 30 cases, and it proved to be nearly impossible to get the suture out without damaging it (Fig. 7).

The aforementioned observations were very enlightening, and they most certainly will be considered in building a new set of prototypes.

Even with the described limitations of the mechanical manipulator, it was shown that an additional number of DOF in an endoscopic instrument is favorable. Although there were no time differences in the experiments, there was a significant difference in the number of actions and failures in the first three experiments. In the laparoscopy group, extra regrasping actions were needed to reposition the coin, rope, or needle inside the grasper tips to be able to fulfil the exercises, leading to extra failures as well. The extra DOF in the mechanical manipulator group facilitated the exercises because the coins, rope, and rings were accessible from different angles. As a result, the participants needed fewer actions and had a smaller number of failures than those in the laparoscopy group.

Considering the modifications to be expected in a next set of mechanical manipulators, it has been shown that the instrument has potential. Along with robotic devices in an experimental or clinical setting, the mechanical manipulator is expected to be a competitive and economical instrument for endoscopic surgery in the near future.

\section{References}

1. Agresta F, Michelet I, Coluci G, Bedin N (2000) Emergency laparoscopy: a community hospital experience. Surg Endosc 14: 484 487

2. Ballantyne GH (2002) Robotic surgery, telerobotic surgery, telepresence, and telementoring: review of early clinical results. Surg Endosc 16: 1389-1402

3. Berguer R, Forkey DL, Smith WD (1999) Ergonomic problems associated with laparoscopic surgery. Surg Endosc Ultras 13: 466468

4. Bodner J, Wykypiel H, Wetscher G, Schmid T (2004) First experiences with the da Vinci operating robot in thoracic surgery. Eur J Cardiothorac Surg 25: 844-851

5. Breedveld P, Wentink M (2000) Eye-hand coordination in laparoscopy: overview of experiments and supporting aids. Minim Invasive Ther Allied Technol 10: 155-162

6. Cadiere GB, Himpens J, Germay O, Izizaw R, Degueldre M, Vandromme J, Capelluto E, Bruyns J (2001) Feasibility of robotic laparoscopic surgery: 146 cases. World J Surg 25: 1467-1477

7. Hubens G, Coveliers H, Balliu L, Ruppert M, Vaneerdeweg W (2003) A performance study comparing manual and robotically assisted laparoscopic surgery using the da Vinci system. Surg Endose 17: 1595-1599

8. Jaspers JE, Den Boer KT, Sjoerdsma W, Bruijn M, Grimbergen CA (2000) Design and feasibility of PASSIST, a passive instrument positioner. J Laparoendosc Adv Surg Tech A 10: 331-335

9. Jaspers JEN, Shehata M, Wijkhuizen F, Herder JL, Grimbergen CA (2004) Mechanical manipulator for intuitive control of endoscopic instruments with seven degrees of freedom. Minim Invasive Ther 13: 191-198

10. Loulmet D, Carpentier A, d'Attellis N, Berrebi A, Cardon C, Ponzio O, Aupecle B, Relland JY (1999) Endoscopic coronary artery bypass grafting with the aid of robotic assisted instruments. J Thorac Cardiovasc Surg 118: 4-10

11. Martin RC II, Kehdy FJ, Allen JW (2005) Formal training in advanced surgical technologies enhances the surgical residency. Am J Surg Aug 190: 244-248

12. Mohr FW, Falk V, Diegeler A, Autschback R (1999) Computer enhanced coronary artery bypass surgery. J Thorac Cardiovasc Surg 117: 1212-1213

13. Nio D, Bemelman WA, Boer KT, Dunker MS, Gouma DJ, Gulik TM (2002) Efficiency of manual versus robotical (Zeus) assisted laparoscopic surgery in the performance of standardized tasks. Surg Endosc 16: 412-415, Epub 16 November 2001

14. Nio D, Diks J, Linsen MA, Cuesta MA, Gracia C, Rauwerda JA, Wisselink W (2005) Robot-assisted laparoscopic aortobifemoral bypass for aortoiliac occlusive disease: early clinical experience. Eur J Vasc Endovasc Surg 29: 586-590

15. Perez A, Zinner MJ, Ashley SW, Brooks DC, Whang EE (2003) What is the value of telerobotic technology in gastrointestinal surgery? Surg Endosc 17: 811-813

16. Prasad SM, Maniar HS, Soper NJ, Damiano RJ Jr, Klingensmith ME (2002) The effect of robotic assistance on learning curves for basic laparoscopic skills. Am J Surg 183: 702-707 
17. Richards KF, Fisher KS, Flores JH, Christensen BJ (1996) Laparoscopic appendectomy: comparison with open appendectomy in 720 patients. Surg Laparosc Endosc 6: 205-209

18. Ruurda JP, Broeders IA, Simmermacher RP, Borel Rinkes IH, Van Vroonhoven TJ (2002) Feasibility of robot-assisted laparoscopic surgery: an evaluation of 35 robot-assisted laparoscopic cholecystectomies. Surg Laparosc Endosc Percutan Tech 12: 41-45

19. Shah PR, Joseph A, Haray PN (2005) Laparoscopic colorectal surgery: learning curve and training implications. Postgrad Med J 81: $537-540$
20. Van Veelen MA (2001) New ergonomic guidelines for laparoscopic instruments. Minim Invasive Ther Allied Technol 10: 163-167

21. Wentink M, Wieringa PA, Jackimowicz JJ, Stultiëns GNM (2000) Quantifying hand-eye coordination problems during minimally invasive surgery: is the surgeon misoriented? Minim Invasive Ther 9: $352-353$

22. Wisselink W, Cuesta MA, Gracia C, Rauwerda JA (2002) Robotassisted laparoscopic aortobifemoral bypass for aortoiliac occlusive disease: a report of two cases. J Vasc Surg 36: 1079-1082 